\title{
VIOLÊNCIA OBSTÉTRICA: CONVERGÊNCIAS E DIVERGÊNCIAS ENTRE ACADÊMICOS DE ENFERMAGEM E MEDICINA
}

Solana Nunes Vieira ${ }^{1}$ Brenda Alice Andrade Vidigal ${ }^{1}$ António Manuel Sousa ${ }^{1}$ Leonardo Naves dos Reis ${ }^{1}$ Elizabeth Teixeira ${ }^{1}$

Milaine Nunes Gomes Vasconcelos ${ }^{1}$

\author{
https://orcid.org/0000-0001-8003-0805 \\ https://orcid.org/0000-0002-6639-2196 \\ https://orcid.org/0000-0002-3347-489X \\ https://orcid.org/0000-0001-6846-7967 \\ https://orcid.org/0000-0002-5401-8105 \\ http://orcid.org/0000-0001-8815-0406
}

Objetivo: Investigar conceitos, compreensão e reconhecimento da violência obstétrica, meios de abordagem da temática dentro da universidade e tipos de violência obstétrica observados, vivenciados e praticados pelos acadêmicos de enfermagem e medicina. Metodologia: Estudo analítico, transversal, com abordagem quantitativa, realizado em uma universidade pública, entre novembro de 2017 a abril de 2018 . A amostra foi de 220 acadêmicos, sendo 76 do curso de enfermagem e 144 do curso de medicina. Os dados foram coletados por meio de questionário. Para análise adotou-se os valores absolutos, percentuais e aplicou-se o teste t de Student para significância estatística. Resultados: Os acadêmicos convergem no que tange ao reconhecimento das agressões psicológicas, verbais e a proibição da presença de acompanhante. Divergem no que se refere à: procedimentos e condutas que para uns é rotina na prática obstétrica, não sendo considerados violência obstétrica; inclusão da temática na grade curricular; discussão da temática em sala de aula. Conclusão: Os acadêmicos apresentaram mais divergências que convergências. Assim, há necessidade de sensibilização e reforço da discussão da temática violência obstétrica.

Descritores: Violência; Obstetrícia; Conhecimento; Universidades.

\section{OBSTETRIC VIOLENCE: CONVERGENCES AND DIVERGENCES BETWEEN NURSING AND MEDICINE ACADEMICS}

Objective: Investigate concepts, understanding and recognition of obstetric violence, means of approaching the subject within the university and types of obstetric violence observed, experienced and practiced by nursing and medical scholars. Methodology: A cross-sectional, analytical study, with a quantitative approach, conducted at a public university between November 2017 and April 2018. The sample consisted of 220 students, of which 76 were nursing students and 144 were medical students. Data was collected through a questionnaire. For the analysis, the absolute and percentage values were adopted and the Student's t-test was applied for statistical significance. Results: The academics converge regarding the recognition of psychological, verbal aggression and the prohibition of the presence of a companion. They differ in respect to: procedures and behaviors that for some are routine obstetric practice, not being considered obstetric violence; inclusion of the theme in the curriculum; discussion of the theme in the classroom. Conclusion: The academics presented more divergences than convergences. Thus, there is a need to raise awareness and reinforce the discussion of obstetric violence.

Descriptors: Violence; Obstetrics; Knowledge; Universities.

\section{VIOLENCIA OBSTÉTRICA: CONVERGENCIAS Y DIVERGENCIAS ENTRE ACADÉMICOS DE ENFERMERÍA Y MEDICINA}

Objetivos: Investigar conceptos, comprensión y reconocimiento de la violencia obstétrica, los medios para abordar el tema dentro de la universidad y los tipos de violencia obstétrica observados, experimentados y practicados por los estudiosos de enfermería y médicos. Metodología: Estudio analítico, transversal, con abordaje cuantitativo, realizado en una universidad pública, entre noviembre de 2017 a abril de 2018. La muestra fue de 220 académicos, siendo 76 del curso de enfermería y 144 del curso de medicina. Los datos fueron recolectados por medio de un cuestionario. Para el análisis se adoptaron los valores absolutos, porcentuales y se aplicó la prueba t de Student para significancia estadística. Resultados: Los académicos convergen en lo que se refiere al reconocimiento de las agresiones psicológicas, verbales y la prohibición de la presencia de acompañante. En lo que se refiere a: procedimientos y conductas que para unos es rutina en la práctica obstétrica, no siendo considerados violencia obstétrica; la inclusión de la temática en la cuadrícula curricular; la discusión de la temática en el aula. Conclusión: Los académicos presentaron más divergencias que convergencias. Así, hay necesidad de sensibilización y refuerzo de la discusión de la temática violencia obstétrica.

Descriptores: La violencia; Obstetricia; Conocimiento; Universidades. 


\section{INTRODUÇÃO}

A violência obstétrica (VO) caracteriza-se como qualquer ato cometido contra a gestante, de natureza física, psicológica, sexual e verbal, exemplificada como a negação de analgesia, o impedimento da entrada do acompanhante durante o parto e pós-parto, uso indiscriminado de procedimentos invasivos, entre outros ${ }^{(1)}$. Assim, toda forma de violência parte do princípio da dominação e da utilização de intervenções desnecessárias ao quadro clínico da parturiente.

Assim, o Ministério da Saúde cria o Programa de Humanização no Pré-Natal e Nascimento (PHPN), através da Portaria no 569/00, que objetiva proporcionar atendimentos com menos intervenções, reduzir o número de óbitos maternos e neonatais e garantir a melhoria no acesso ao serviço de saúde ${ }^{(2)}$. Apesar da criação de programas que adequem o atendimento, a formação dos profissionais da área da saúde ainda é insuficiente ${ }^{(3)}$

Sendo a Universidade responsável pela formação profissional, de forma crítica e técnica, há a necessidade de adequar o ensino ao que é preconizado pelos programas do Ministério da Saúde e embasado em estudos atuais. No entanto, visualizam-se alunos utilizando como material de estudo o ser humano, expondo a intimidade de mulheres em momento de parto, desconsiderando valores culturais, emocionais, ferindo o princípio ético profissional e comumente apoiado por seus preceptores. A ausência do ensino sobre políticas de humanização do parto e nascimento nas universidades torna o atendimento mais despersonalizado e desumanizado(4).

É nesse contexto que a educação se torna fundamental para desmistificar um ciclo sociocultural pré-estabelecido durante anos, para que atos intimidadores, humilhantes e repugnantes não se tornem impunes no âmbito hospitalar ${ }^{(5)}$. Por isso, a importância da inclusão na universidade, de práticas baseadas em evidências científicas que possam contribuir na redução de técnicas utilizadas como rotina, as quais muitas vezes, são classificadas como VO(3).

A pesquisa torna-se relevante por conhecer a realidade da VO, vivenciada pelos alunos, seja em sala de aula ou em campo de prática, pois a universidade é o caminho para que a mudança deste cenário ocorra. Além disso, existe a necessidade de buscar mais conhecimentos e divulgação sobre a realidade da VO sofrida durante a gestação e parturição, que são cometidas por profissionais de diversas áreas.

Assim, o presente artigo teve por objetivo investigar conceitos, compreensão e reconhecimento da VO, meios de abordagem da temática dentro da universidade e tipos de violência obstétrica observados, vivenciados e praticados pelos acadêmicos de enfermagem e medicina.

\section{METODOLOGIA}

\section{Tipo de estudo}

Trata-se de um estudo analítico, transversal, com abordagem quantitativa.

\section{Participantes da pesquisa}

A população foi composta por acadêmicos de enfermagem e medicina, possuindo como critério de inclusão ter cursado, nos períodos de 2016/1, 2016/2 e 2017/1 as disciplinas Processo de Enfermagem no Cuidar em Saúde da Mulher e Ginecologia e Obstetrícia para os respectivos cursos, resultando em 94 acadêmicos de Enfermagem e 228 acadêmicos de Medicina. No tocante aos critérios de exclusão, não foram incluidos no estudo os alunos que, no momento da coleta de dados, estavam cursando a disciplina Estágio Rural, considerando a dificuldade de acesso às áreas rurais no contexto amazônico.

Para cálculo amostral, baseou-se na amostragem estratificada, considerando o Intervalo de Confiança (IC) de 95\%, resultando em 76 alunos de Enfermagem e 144 alunos de Medicina. Por meio de amostragem aleatória simples realizou-se sorteio das matrículas dos alunos de cada período, para posteriori identificação e contato, apresentação do projeto e solicitação de participação, sendo realizado a assinatura do Termo de Consentimento Livre e Esclarecido.

\section{Local de estudo}

Realizado na Escola Superior de Ciências da Saúde da Universidade do Estado do Amazonas, no período de novembro de 2017 a abril de 2018

\section{Coleta de dados}

Para coleta de dados, aplicou-se um questionário pré-elaborado pelos pesquisadores, com variáveis sociodemográfica; período que cursou as disciplinas; conceitos, compreensão e reconhecimento da VO (área 1); meios de abordagem da temática na universidade (área 2); os tipos de violência obstétrica observados, vivenciados e praticados pelos acadêmicos (área 3).

O instrumento utilizado, continha, nas áreas 1 e 2, foi composto por 11 e 13 questões em formato de escala Likert, cujos escores podem variar de 1 a 5, conforme o grau de concordância com afirmação exposta, em que 1 equivale a inadequado e 5 adequado. Já para a área 3, o instrumento continha 10 questões, sendo que em 4 destas, o participante deveria responder sim ou não à pergunta; em outras 4 , deveria escolher a resposta entre alternativas que the eram colocadas; e as outras 2 eram perguntas abertas. 


\section{Procedimento de análise dos dados}

Para mensurar a adequação de conhecimentos e da abordagem da temática na grade curricular (áreas 1 e 2) utilizou-se as médias de pontuação conforme as áreas, efetuando-se a comparação entre os grupos (medicina e enfermagem) por meio do teste $t$ de student, por meio do software IBM SPSS20.

Para a área 3, foi utilizado a análise em valores absolutos e percentuais. Os dados foram organizados em planilha e analisados no programa Microsoft Excel 2013, apresentados por meio de tabelas e gráficos, em valores absolutos, percentuais e por nível descritivo.

\section{Procedimentos éticos}

O projeto seguiu as normas da Resolução CNS 466/12(6), com aprovação do Comitê de Ética em Pesquisa da Universidade do Estado do Amazonas sob parecer no 2.227.100. Todos os participantes assinaram o Termo de Consentimento Livre e Esclarecido do qual thes foi entregue uma cópia.

\section{RESULTADOS}

Os acadêmicos foram predominantemente jovens $(51,8 \%)$, do sexo feminino $(52,2 \%)$, procedentes do interior (43,1\%), e (39,0\%) tinham cursado a disciplina Saúde da Mulher/Ginecologia e Obstetrícia no período 2017.1 (Tabela 1).

Tabela 1 - Caracterização dos acadêmicos do curso de enfermagem e medicina, que cursaram a disciplina Saúde da Mulher/Ginecologia e Obstetrícia. Manaus (AM), Brasil, 2017-2018

\begin{tabular}{|c|c|c|c|c|}
\hline \multirow{2}{*}{ Variáveis } & \multicolumn{2}{|c|}{ Enfermagem } & \multicolumn{2}{|c|}{ Medicina } \\
\hline & $\mathbf{N}$ & $(\%)$ & $\mathbf{N}$ & (\%) \\
\hline \multicolumn{5}{|l|}{ Faixa Etária } \\
\hline 18 a 24 anos & 52 & 68,4 & 62 & 43,1 \\
\hline 25 a 29 anos & 15 & 19,7 & 49 & 34,0 \\
\hline 30 anos ou mais & 9 & 11,8 & 28 & 19,4 \\
\hline Sem informação & - & - & 5 & 3.5 \\
\hline \multicolumn{5}{|l|}{ Sexo } \\
\hline Feminino & 51 & 67,1 & 64 & 44.4 \\
\hline Masculino & 25 & 32,9 & 79 & 54.9 \\
\hline Sem informação & - & - & 1 & 0,7 \\
\hline \multicolumn{5}{|l|}{ Procedência } \\
\hline Capital & 27 & 35,5 & 65 & 45,1 \\
\hline Interior & 43 & 56,6 & 52 & 36,1 \\
\hline Sem informação & 6 & 7,9 & 27 & 18,8 \\
\hline \multicolumn{5}{|l|}{ Periodo } \\
\hline 2016.1 & 23 & 30,0 & 32 & 22,2 \\
\hline 2016.2 & 25 & 33,0 & 54 & 37,5 \\
\hline 2017.1 & 28 & 37,0 & 58 & 40,3 \\
\hline
\end{tabular}

Sobre conceitos, compreensão e reconhecimento de VO (área 1), foi evidenciado a média quatro entre os estudantes. Quando comparado os dois cursos, foi observado maior representatividade percentual, nas classificações satisfatório e adequado, nos alunos do curso de enfermagem. (Gráfico 1).

Gráfico 1: Percentual de adequação, quanto ao conhecimento sobre Violência Obstétrica entre os acadêmicos. Manaus (AM), Brasil, 2017-2018.

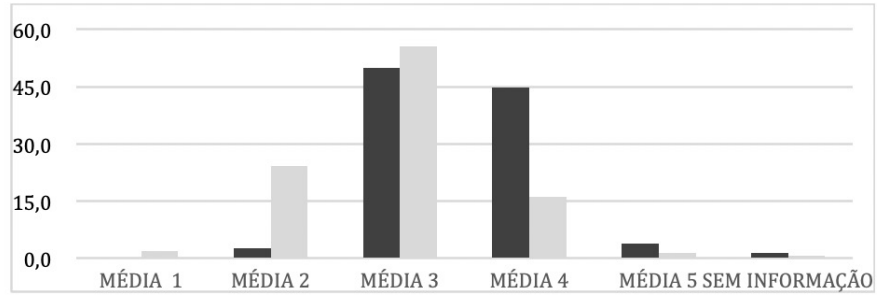

Para os alunos dos cursos de Enfermagem 63 (82,9\%) e Medicina 94 (64,3\%), a universidade foi o local de primeiro contato com a temática violência obstétrica. Outros locais foram citados pelos acadêmicos, como: ambiente familiar oito (10,5\%) e 14 (9,7\%); e o ambiente hospitalar cinco (6,6\%) e 27 (18,8\%), respectivamente.

Sobre os meios de abordagem da temática na universidade (área 2), foi evidenciada a média três entre os acadêmicos. Observou-se que os alunos de enfermagem se mantiveram entre a imparcialidade e satisfeitos quanto à avaliação da inclusão da temática no seu curso. Enquanto na medicina, houve predominância para a permanência da imparcialidade, seguido da insatisfação. (Gráfico 2).

Gráfico 2: Percentual da avaliação dos alunos sobre a inserção da temática Violência Obstétrica na grade curricular, nos cursos de enfermagem e medicina. Manaus (AM), Brasil, 2017-2018.

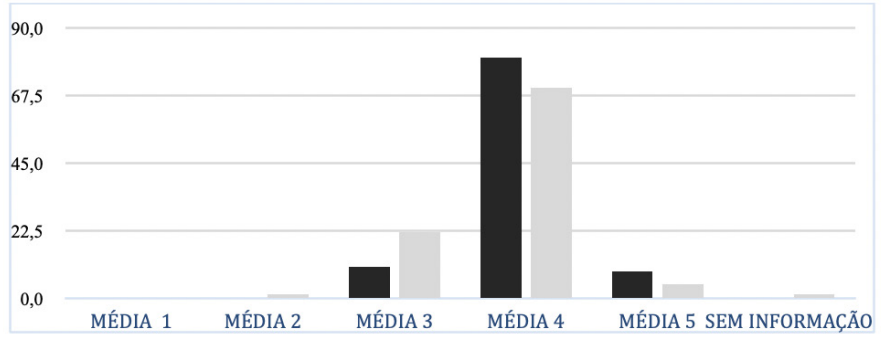

Ao comparar a diferença das médias e desvios-padrões dos dois cursos, foi observado nas áreas 1 e 2, diferença significativa do ponto de vista estatístico. Na área 1, apesar de menor diferença de média entre os cursos, os acadêmicos de enfermagem mostraram-se mais coesos no 
que se refere à conhecimentos sobre VO, comparado aos acadêmicos de medicina. Na área 2, foi constatado maior diferença entre as médias, porém mais similaridade entre os grupos, e os acadêmicos mostraram-se mais imparciais quanto a avaliação da inserção da temática na grade curricular (Tabela 2).

Tabela 2 - Comparação das médias dos acadêmicos quanto ao conhecimento (área l) e inserção na grade curricular (área 2) da temática violência obstétrica. Manaus (AM), Brasil, 2017-2018.

\begin{tabular}{|c|c|c|c|c|c|c|}
\hline Área & Curso & $\mathrm{N}$ & $\begin{array}{l}\text { Média } \\
\text { (dp) }\end{array}$ & $\begin{array}{l}\text { Diferença } \\
\text { de médias }\end{array}$ & $\begin{array}{l}\text { p-va- } \\
\text { lor* }\end{array}$ & IC $95 \%$ \\
\hline \multirow{2}{*}{1} & Enfermagem & 76 & $4,5(0,40)$ & \multirow{2}{*}{0.31} & \multirow{2}{*}{$\begin{array}{l}<0.01 \\
0.48)\end{array}$} & \multirow[t]{2}{*}{ (0.13; } \\
\hline & Medicina & 144 & $4,2(0,71)$ & & & \\
\hline \multirow{2}{*}{2} & Enfermagem & 76 & $3.8(0,60)$ & \multirow{2}{*}{0.50} & \multirow{2}{*}{$\begin{array}{l}<0.01 \\
0.69)\end{array}$} & \multirow[t]{2}{*}{$(0,31$} \\
\hline & Medicina & 144 & $3.3(0,70)$ & & & \\
\hline
\end{tabular}

*teste-t de Student.

As práticas consideradas como violentas, pelos acadêmicos de enfermagem e medicina respectivamente, foram: agressões psicológicas 74 (97,4\%) e 135 (93,8\%), proibir a presença do acompanhante 70 (92,1\%) e 109 (75,7\%), episiotomia 63 (82,9\%) e 17 (11,8\%), manobra de Kristeller 61 $(80,3 \%)$ e 49 (34,0\%), e exame tocológico a cada 4 horas 30 $(39,5 \%)$ e cinco $(3,5 \%)$.

Referente à vivência de VO pelos acadêmicos de enfermagem e medicina, respectivamente, 45 (59,2\%) e 55 $(38,2 \%)$ afirmam que já presenciaram alguma atitude violenta por parte dos profissionais, sendo as mais observadas: as agressões verbais 30 (66,7\%) e 45 (81,8\%), episiotomia $13(28,9 \%)$ e cinco (9,1\%), manobra de Kristeller 11 $(24,4 \%)$ e sete $(12,7 \%)$, proibição da presença do acompanhante $10(22,2 \%)$ e seis $(10,9 \%)$.

A realização de práticas de VO pelos alunos de enfermagem foi pouco evidenciada quando a maioria 68 (89,5\%) afirmou nunca ter praticado, seis $(7,9 \%)$ praticaram pelo menos uma vez, um $(1,3 \%)$ praticou de duas a três vezes, e um (1,3\%) não informou. Entre os acadêmicos de medicina a evidência foi similar, pois 139 (96,5\%) afirmaram nunca terem realizado $\mathrm{VO}$, dois $(1,4 \%)$ afirmaram a realização de pelo menos uma vez, um $(0,7 \%)$ quatro vezes ou mais e dois $(1,4 \%)$ não informaram.

As ações pedagógicas utilizadas e que contribuíram para formação dos acadêmicos sobre a temática estudada, foram identificadas respectivamente como: orientações em sala de aula 46 (60,5\%) e 62 (43,1\%), participação de palestras, simpósios e rodas de conversas 26 (34,2\%) e 26
(18,1\%), e aulas práticas em maternidades 12 (15,8\%) e 41 (28,5\%). Foi observado ainda, que entre os alunos de medicina, 23 (16,0\%) referiram não haver momentos de discussão da temática em sala de aula, sete (4,9\%) consideraram que os materiais de estudo eram atualizados; e um (0,7\%) referiu que o tema não havia sido abordado, e que apenas aprenderam sobre manobras a serem utilizadas na condução do trabalho de parto.

A temática VO inserida no ambiente acadêmico proporcionou mudanças conceituais e comportamentais referidas por $88,2 \%$ dos alunos de enfermagem e $68,8 \%$ dos alunos de medicina. No curso de enfermagem, 44 (65,7\%) dos acadêmicos passaram a entender sobre os direitos da mulher, respeitar suas decisões, além de ofertar boas práticas durante o trabalho de parto; 11 (16,4\%) perceberam a necessidade de realizar melhor acolhimento e preservar os direitos das mulheres; seis (9,0\%) compreenderam melhor a definição ou redefinição da VO; dois (3,0\%) passaram a ver procedimentos que considerava normal, como VO; dois (3,0\%) sem informação.

Para os acadêmicos de medicina, 41 (41,4\%) passaram a entender os direitos e respeitar as decisões da parturiente; 17 (17,2\%) entenderam que a mulher deve ser melhor acolhida e conseguiram definir ou redefinir VO; nove $(9,1 \%)$ afirmaram que a temática não foi abordada, não havendo referências sobre mudanças; seis $(6,1 \%)$ perceberam que procedimentos realizados na obstetrícia como rotina, são VO; quatro (4,0\%) afirmaram haver a necessidade de definir até onde a parturiente pode decidir no momento do parto, além de desconsiderar a associação da violência à assistência médica; e quatro (4,0\%) sem informação.

\section{DISCUSSÃO}

O estudo evidenciou que a população jovem é predominante nos dois cursos. Entre 2004 a 2014, o crescimento da inserção de jovens, no ensino superior, entre 18 e 24 anos, foi cerca de $58,5 \%{ }^{(7)}$. Assim, o fato de ser jovem e a ausência de vivências com a temática podem influenciar no conhecimento e/ou desconhecimento da mesma, refletindo no amadurecimento profissional. Além disso, a universidade é corresponsável por um ensino diversificado e de excelência, embasados em evidências atuais.

O conhecimento de assuntos atuais e a sua abordagem no espaço acadêmico, é fundamental para reflexão quanto ao processo de formação profissional, transformando e melhorando conceitos e condutas para uma assistência mais humana e respeitosa ${ }^{(8)}$. Entre esses assuntos, a VO torna-se extremamente relevante para abordagem e discussão no meio acadêmico, justamente por ser na gradua- 
ção, o início da preparação e formação dos futuros profissionais, podendo assim contribuir para mudança do cenário obstétrico atual(3).

Entre os cursos estudados, a enfermagem apresentou maior contato com a temática VO em sala de aula. Talvez por este curso enfatizar a importância do cuidado e da humanização em sua assistência. Estudos indicam a necessidade da abordagem sobre a humanização, e consequentemente, o direcionamento para a VO, com foco na formação profissional adequada e aplicação do conhecimento em ambiente de trabalho e/ou campo de aula prática, apesar da implantação do cuidado humanizado estar sendo feito com dificuldade e de forma lenta(8-9)

No curso de Medicina, por ser tradicionalmente pautado pelo modelo biomédico, apresenta a necessidade da inclusão de algumas temáticas em sua grade curricular que permitam conhecimentos baseados em evidências científicas, proporcionando ao acadêmico uma visão e decisão crítica com relação a melhor conduta a ser tomada na assistência à mulher. Inclusive para mudanças em condutas e comportamentos que, no momento tão particular como o trabalho de parto e parto, acabam prevalecendo habilidades técnicas, tornando a mulher apenas um objeto de estudo(3,6)

A ausência de discussão, a insatisfação, acreditar que o assunto seja irrelevante para formação profissional, ou mesmo a abordagem não efetiva do assunto pelos professores, são fatores que induzem o desinteresse dos acadêmicos sobre o tema ${ }^{(3)}$. Além disso, existe a necessidade de relacionar o ensino humanístico ao ensino biomédico, modificando a prática assistencial na hora do parto ${ }^{(10)}$. No presente estudo, apesar de ter havido mudanças em conceitos e comportamentos na maioria dos estudantes, ainda foi representativo a imparcialidade dos acadêmicos de medicina quanto à inclusão da temática na grade curricular. Este fato desperta reflexão sobre as estratégias para a abordagem da temática pelo curso.

O curso de Enfermagem prioriza e incentiva as boas práticas seguindo evidências científicas, protocolos do Ministério da Saúde, como o programa de humanização, parto e nascimento; visando formação profissional para assistência de qualidade e humanizada, contribuindo para diminuição de ocorrências de $\mathrm{VO}^{(2,8)}$. Corroborando com os autores, foi evidenciado diferença significativa do ponto de vista estatístico, mostrando a enfermagem mais coesa quanto ao conhecimento da temática estudada, e satisfação quanto a inclusão na grade curricular.

A ocorrência de VO é uma prática muito comum em todo o processo gestacional, de parturição e puerperal. Estudo realizado em Recife em 2016 evidenciou que a maio- rias das puérperas tinham sofrido algum tipo de violência durante o parto ${ }^{(11)}$. Realidade identificada, no presente estudo, de forma divergente entre a enfermagem e a medicina. A enfermagem reconhece, em sua maioria, a ocorrência e a vivência dessa prática violenta. Porém percebe-se dificuldade de reconhecimento na medicina, quando a maioria dos acadêmicos tratam alguns procedimentos utilizados como rotineiro.

As agressões verbais são caracterizadas como VO, onde os profissionais usam termos depreciativos, comentários irônicos, piadas sobre comportamento da mulher e ameaças durante o parto(12). Além de práticas verbais, ações técnicas, sem indicação para utilização, torna-se uma violência. Exemplificando, a episiotomia é um procedimento que vem sendo abolido por muitos autores ${ }^{(13-14)}$. Porém, de acordo com o inquérito Nascer no Brasil o procedimento foi realizado em $53,5 \%$ dos partos ${ }^{(15)}$. Essas práticas sejam no momento do pré-natal, parturição ou puerperal, foram evidenciadas por acadêmicos de enfermagem, em Teresina ${ }^{(16)}$. Corroborando com os autores citados, as agressões verbais e a episiotomia ainda são frequentes, e foram evidenciadas pelo estudo como as práticas mais realizadas em campo de estágio.

O toque vaginal avalia a dilatação do colo uterino e evidencia a evolução do trabalho de parto. Preconiza-se a realização do procedimento a cada 4 horas durante o primeiro período do parto, sempre após o consentimento da parturiente ${ }^{(17)}$. Porém, o que ocorre é a realização em intervalos menores e por diversos profissionais, expondo a mulher ao procedimento desnecessariamente e sem o consentimento da mesma(18).

Outra forma de VO considerada pelos acadêmicos, porém pouco observada nas maternidades, é a proibição da presença do acompanhante. De acordo com a Lei no 11.108 de 7 de abril de 2005, a parturiente tem direito à escolha de um acompanhante, em todo o trabalho de parto e pós- parto ${ }^{(19)}$. Por ter sido assegurado por lei esse direito, qualquer forma de ignorá-lo se configura uma violência. Mesmo após dez anos da vigência da lei, ainda existem locais que privam as mulheres da presença do acompanhante de escolha, seja na fase de trabalho de parto, parto ou puerpério, afirmando "não ser permitido"(20). Outros estudos mostram melhor adesão ao direito da mulher, trazendo a presença do acompanhante, como boa prática durante o trabalho de parto(15,20).

Abordagem de temáticas novas e que estimulem análise crítica refletindo em mudanças de conceitos, comportamentos e práticas, se torna um desafio a ser enfrentado pela universidade. $\mathrm{O}$ aprendizado dos acadêmicos, baseado 
em evidências, propostas ativas, no holístico e na empatia, pode ser determinante na construção do perfil profissional. Estudos apontam as metodologias ativas como formas de aprendizado eficiente, promoção da autonomia profissional, visão crítica para avaliação clínica e tomada de decisões mais coerentes, o que na obstetrícia deve ser embasado e determinado pela real necessidade do procedimento, para evitar possiveis complicações. As metodologias ativas não se limitam pela busca do conhecimento apenas do acadêmico, mas o conjunto (universidade, professor e aluno) trabalham para que a metodologia seja executada de maneira proveitosa e efetiva. Com essa aplicabilidade, espera-se que mudanças efetivas aconteçam nas atitudes e comportamentos, como no cenário de VO, visto como ações que deixam consequências físicas e psicológicas nas mulheres ${ }^{(3-5)}$. Na prática, a humanização, amplamente discutida desde a década de 80 , enfrenta situações que acaba por dificultar a sua total implantação e redução na ocorrência de violência obstétrica(21). Porém, quando essas mudanças ocorrem, são perceptiveis nas afirmações de mulheres sobre a experiência do parto ser positiva, humana e respeitosa, por meio da assistência integral de equipe multidisciplinar, que acolhe e auxilia todo o processo desde a admissão até a alta hospitalar. Além disso, a forte contribuição para a redução da ansiedade e dos medos oriundos desse momento único na vida de cada mulher(22).

\section{Limitações do estudo}

O estudo teve como limitações a dificuldade de aplicação do instrumento com os acadêmicos de medicina, por alguns estarem no período em que as aulas são exclusivamente em ambiente hospitalar, além da dificuldade dos acadêmicos em relatar as próprias vivências sobre violência obstétrica. Apesar disso, foi alcançado a amostra proposta no estudo. Ressalta-se ainda, a escassez de publicações que referenciem o cenário acadêmico sobre a temática.

\section{Contribuição do estudo para a prática}

Espera-se que a pesquisa possa contribuir para reflexão do cenário acadêmico, promover mudanças de postura profissional, do ensino de forma tecnicista e do atual cenário obstétrico. Mudanças fundamentais para melhoria da qualidade da educação, da assistência humanizada e a garantia de direitos da mulher a um parto sem violência.

\section{CONCLUSÃO}

Os acadêmicos apresentaram um conhecimento satisfatório sobre VO, seja por vivência nas maternidades e/ou orientações em sala de aula. Porém, houve conhecimentos e compreensões divergentes entre os dois cursos quando questionados sobre assuntos direcionados a alguns procedimentos e condutas vistos como rotinas, não sendo considerados como VO; à inclusão da temática na grade curricular, e à discussão da temática em sala de aula. Nessa conjuntura, percebeu-se a importância da inclusão e discussão da temática na universidade, por meio de metodologias ativas e efetivas, para contemplar e sensibilizar o maior número de acadêmicos possiveis, visando mudanças de conceitos, comportamentos e práticas.

\section{CONTRIBUIÇÕES DOS AUTORES}

SNV e MNGV: concepção e/ou desenho, análise e interpretação dos dados, redação do artigo, revisão crítica, revisão final. BAAV: concepção e/ou desenho, análise e interpretação dos dados, redação do artigo. MAS e LNR: análise e interpretação dos dados, revisão final. ET: revisão crítica, revisão final.

\section{REFERÊNCIAS}

1.Martins AC, Barros GM. Will you give birth in pain? Integrative review of obstetric violence in Brazilian public units. Rev Dor [Internet]. 2016 [cited 2018 June 13]; 17(3): 215-8. Available from: http://www.scielo.br/pdf/rdor/v17n3/1806-0013-rdor-17-03-0215. pdf

2. Ministério da Saúde (BR). Gabinete do Ministro. Portaria no 569 de 1 ○ de Junho de 2000. Instituir o Programa de Humanização no Pré-natal e Nascimento, no âmbito do Sistema Único de Saúde [Internet]. Brasilia: Ministério da Saúde: 2000 [cited 2018 Mar 21]. Available from: http://bvsms.saude.gov.br/bvs/saudelegis/ gm/2000/prt0569_01_06_2000_rep.html

3. Souza AL, Silva LC, Alves RN, Alarcão ANJ. Factors associated

with obstetric violence: An integrative review of the literature. Rev ciênc méd [Internet]. 2016 [cited 2018 June 15]: 25(3):115-28. Available from: https://seer.sis.puc-campinas.edu.br/seer/index. $\mathrm{php/cienciasmedicas/article/view/3641/2486}$

4. Diniz CSG, Niy DY, Andresso HFA, Carvalho PCA, Salgado HO. The vagina-school: interdisciplinary seminar on violence against woman in the teaching of the health professions. Interface comun saúde educ [Internet]. 2016 [cited 2018 Aug 15]; 20(56):253-9. Available from: http://www.scielo.br/pdf/icse/v20n56/1807-5762icse-20-56-0253.pdf

5. Diniz SG, Salgado HO, Andrezzo HFA, Carvalho PGC, Carvalho PCA, Aguiar CA, et al. Abuse and disrespect in childbirth care as 
a public health issue in Brazil: origins, definitions, impactas on maternal health, and proposals for its prevention. J Hum Growth Dev [Internet]. 2015 [cited 2018 July 29]; 25(3):377-84. http:// pepsic.bvsalud.org/pdf/rbcdh/v25n3/19.pdf

6. Ministério da Saúde (BR). Conselho Nacional de Saúde. Resolução no 466, de 12 de dezembro de 2012. Aprova diretrizes e normas regulamentadoras de pesquisas envolvendo seres humanos [Internet]. Brasilia: Ministério da Saúde; 2012 [cited 2018 June 18]. Available from: http://bvsms.saude.gov.br/bvs/saudelegis/ cns/2013/res0466_12_12_2012.html

7. Sposito MP, Souza R, Silva FA. The research on young people in Brazil: setting new challenges from quantitative data. Educ Pesqui [Internet]. 2018 [cited 2018 July 18]; 44:e170308. Available from: http://www.scielo.br/pdf/ep/v44/en_1517-9702ep-S1678-4634201712170308.pdf

8. Barbosa GC, Meneguim S, Lima SAM, Moreno V. National Policy of Humanization and education of health care professionals: integrative review.. Rev Bras Enferm [Internet]. 2013 [cited 2018 June 18]: 66(1):123-7. Available from: http://www.scielo.br/pdf/ reben/v66nl/v66nlal9.pdf

9. Pasche DF, Vilela MEA, Giovanni MD, Almeida PVB, Netto TLF. Rede Cegonha: desafios de mudanças culturais nas práticas obstétricas e neonatais. Divulg saúde debate [Internet]. 2014 [cited 2018 June 15]; (52):58-71. Available from: http://portalarquivos2. saude. gov.br/images/pdf/2015/maio/15/1.b\%20-\%20Divulgacao-52. pdf $\#$ page $=60$

10. Possati AB, Prates LA, Cremonese L, Scarton J, Alves CN, Ressel LB. Humanization of childbirth: meanings and perceptions of nurses. Esc Anna Nery Rev Enferm [Internet]. 2017 [cited 2018 July 14]; 21(4):1-6. Available from: http://www.scielo.br/pdf/ean/ v2ln4/1414-8145-ean-2177-9465-EAN-2016-0366.pdf

11. R10. ebello MTMP, Rodrigues Neto JF. The humanization of childbirth care from the perspective of medical students. Rev bras educ med [Internet]. 2012 [cited 2018 June 15]: 36(2):188-97. Available from: http://www.scielo.br/pdf/rbem/v36n2/06.pdf

12. Palma CC, Donelli TMS. Obstetric violence in Brazilian womans. Rev Psico (Porto Alegre) [Internet]. 2017 [cited 2018 June 28]; 48(3):216-30. Available from: http://revistaseletronicas.pucrs.br/ ojs/index.php/revistapsico/article/view/25161/pdf

13. Santos LM, Santos LMS, Brandão MM, Cerqueira EAC, Ramos MSX, Carvalho ESS. Association between perineorrhaphy and perineal problems, habitual activities and physiological needs affected. Rev Cuid [Internet]; 2018 [cited 2018 June 25]; 9(2):223344. Available from: https://www.revistacuidarte.org/index.php/ cuidarte/article/view/530/964

14. Costa ML, Pinheiro NM, Santos LFP, Costa SAA, Fernandes AMG. Episiotomia no parto normal: incidência e complicações. Carpe Diem [Internet]. 2015 [cited 2018 Jan 22]: 13(1): 173-87. Available from: https://periodicos.unifacex.com.br/Revista/article/ view/655/pdf
15. Ministério da Saúde (BR). Fundação Oswaldo Cruz. Nascer no Brasil: Inquérito Nacional sobre Parto e Nascimento [Internet]. Brasilia: Ministério da Saúde: 2014 [cited 2018 June 25]. Available from: http://www6.ensp.fiocruz.br/nascerbrasil/

16. Costa FL, Cintra HMP, Azevedo FHC. Perception of Nursing Academics on Obstetric Violence. Rev Saúde Foco [Internet]. 2017 [cited 2018 Aug 02]; 4(2):71-103. Available from: http:// www4.fsanet.com.br/revista/index.php/saudeemfoco/article/ view/1508/491491504

17. Ministério da Saúde (BR). Secretaria de Ciência, Tecnologia e Insumos Estratégicos, Departamento de Gestão e Incorporação de Tecnologias em Saúde. Diretrizes nacionais de assistência ao parto normal: versão resumida [Internet]. Brasilia: Ministério da Saúde: 2017 [cited 2018 Aug 28]. Available from: http://bvsms. saude.gov.br/bvs/publicacoes/diretrizes_nacionais_assistencia_ parto_normal.pdf

18. Biscegli TS, Grio JM, Melles LC, Ribeiro SRMI, Gonsaga RAT. Obstetrical violence: profile assistance of a state of São Paulo interior maternity school. CuidArte Enferm [Internet] 2015 [cited 2018 June 15]; 9(1):18-25. Available from: http://fundacaopadrealbino.org.br/facfipa/ner/pdf/ Revistacuidarteenfermagem\%20v.\%209\%20n.1\%20\%20jan.\%20 jun\%202015.pdf

19. Brasil. Lei 11.108 , de 08 de abril de 2005. Altera a Lei no 8.080 para garantir às parturientes o direito à presença de acompanhante durante o trabalho de parto, parto e pós-parto. Diário Oficial da União [Internet]. 2005 Apr 08 [cited 2018 June 19]; Seção 1. p. 1. Available from: http://www.planalto.gov.br/ cciVil_03/_Ato2004-2006/2005/Lei/L11108.htm

20. Gonçalves AC, Rocha CM, Gouveia HG, Armellini CJ, Moretto $\mathrm{VL}$, Moraes BA. The companion in the obstetrics centre of a university hospital in southern Brazil. Rev Gaúcha Enferm [Internet]. 2015 [cited 2018 July 15]; 36(Spe):159-67. Available from: http://www.scielo.br/pdf/rgenf/v36nspe/0102-6933-rgenf36-spe-0159.pdf

21. Pinto LMTR, Carvalho JSN, Correia RM, Lins ESF, Oliveira LLF, Santos AAP. Interfaces entre profissionais de saúde e a humanização da assistência ao parto. Revista Enfermagem em Foco [Internet]. 2018 [cited 2019 Feb 06]; 9 (3): 53-58. Available from: http://revista.cofen.gov.br/index.php/enfermagem/article/ view/1195/461

22. Barros FRB, Accioly LM, Freitas WFM, Andrade LL, Silva BKC, Araújo RO. Percepção das puérperas manauaras frente à assistência de enfermagem no preparo do trabalho de parto e nascimento. Revista Enfermagem em Foco [Internet]. 2018 [cited 2019 Feb 06]: 9 (1): 76-81 Available from: http://revista.cofen.gov. br/index.php/enfermagem/article/view/1035/432

RECEBIDO: 04/11/2018

ACEITO: 23/09/2019 